\title{
A REMARK ON PRÜFER RINGS
}

\author{
EDWARD D. DAVIS ${ }^{1}$
}

An integral domain $R$ is called a Prüfer ring if for every maximal ideal $M$ of $R, R_{M}$ is a valuation ring. The following theorem embodying a new characterization of this class of rings came to light in considering a question of extending valuations (see Corollary 3). Other characterizations of Prüfer rings due to Hattori [1] and Richman [2] are immediate consequences of this new one (see Corollaries 1 and 2). In what follows all rings are commutative with $1 \neq 0$.

THEOREM. Let $R$ be an integral domain with quotient field $K$. Then the following statements are equivalent.

(1) $R$ is a Prüfer ring.

(2) Given $K$-algebras $\mathfrak{A}$ and $\mathfrak{B}$ and $R$-subalgebras $A$ of $\mathfrak{A}$ and $B$ of $\mathfrak{B}$, then the canonical homomorphism $A \otimes_{R} B \rightarrow \mathfrak{A} \otimes_{K} \mathfrak{B}$ is injective.

(3) The tensor product of any two $R$-subalgebras of $K$ is an integral domain.

CoRollary 1 (Hattori [1]). $R$ is a Prïfer ring if the tensor product of torsion-free $R$-modules is torsion-free.

Corollary 2 (RIChman [2]). $R$ is a Prïfer ring if every $R$-subalgebra of $K$ is a flat $R$-module.

Corollary 3. Let $\mathfrak{A}, \mathfrak{B}$ and $K$ be subfields of a field $L$ with $\mathfrak{A}$ and $\mathfrak{B}$ intermediate between $K$ and $L$ and linearly disjoint over $K$. Suppose $A$ and $B$ are valuation rings of $\mathfrak{A}$ and $\mathfrak{B}$ respectively such that $A \cap K$ $=B \cap K$. Then there is a valuation ring of $L$ dominating both $A$ and $B$.

REMARKs. 1. The characterization of Dedekind rings provided by the theorem is also new. 2. In the course of the proof we shall see that (3) can be replaced by an ostensibly weaker condition: Given nonzero $a$ and $b$ in $R$, then $b$ is not a divisor of zero in $R[a / b] \otimes R[b / a]$. 3. The hypotheses of Corollaries 1 and 2 are well-known properties of Prüfer rings (see, for example, $\$ 4$ of Chapter VII in Cartan and Eilenberg [3]).

Proof of Corollaries 1 and 2 . Let $A$ and $B$ be $R$-subalgebras of $K$ and let $S=R-\{0\}$. If $A \otimes_{R} B$ is torsion-free, then $A \otimes_{R} B$ is a subring of $\left(A \otimes_{R} B\right)_{S}=A_{S} \otimes_{R_{S}} B_{S}=K \otimes_{K} K=K$. If $B$ is $R$-flat, then

Received by the editors September 8, 1967.

${ }^{1}$ Supported in part by the National Science Foundation under grant GP-6388. 
$A \otimes_{R} B$ is a subring of $K \otimes_{R} B=K$. That is, under the hypothesis of either corollary, $A \otimes_{R} B$ is an integral domain.

Proof of Corollary 3. Let $R=A \cap K=B \cap K$. We are given that the homomorphism $\mathfrak{A} \otimes_{K} \mathfrak{B} \rightarrow L$ (such that $\left.a \otimes b \leftrightarrow a b\right)$ is injective. Consequently, in view of the equivalence of (1) and (2), we know that the induced homomorphism $A \otimes_{R} B \rightarrow L$ is an isomorphism of $A \otimes_{R} B$ with the compositum $C$ of $A$ and $B$ in $L$. Let $\bar{A}, \bar{B}$ and $\bar{R}$ denote the residue fields of $A, B$, and $R$ respectively. The homomorphism $A \otimes_{R} B \rightarrow \bar{A} \otimes_{\bar{R}} \bar{B}$ given by $a \otimes b \mapsto \bar{a} \otimes \bar{b}$ induces a homomorphism of $C$ with kernel a proper ideal containing the maximal ideals of $A$ and $B$. Let $M$ be any maximal ideal of $C$ containing this kernel. Then any valuation ring of $L$ dominating $C_{M}$ is a desired extension.

Proof of the Theorem. (1) $\rightarrow(2)$. Since $A$ and $B$ are torsion-free $R$-modules, so is $A \otimes_{R} B$. (This well-known property of modules over Prüfer rings follows easily from the simple observation that torsionfree modules of finite type over valuation rings are free.) Let $S$ $=R-\{0\}$. Then $A \otimes_{R} B$ injects canonically into $\left(A \otimes_{R} B\right)_{S}$ $=A_{S} \otimes_{R_{S}} B_{S}=A_{S} \otimes_{K} B_{S}$ which injects canonically into $\mathfrak{A} \otimes_{K} \mathfrak{B}$. $(2) \rightarrow(3)$ is clear. In order to establish (3) $\rightarrow(1)$ we introduce a fourth statement, not involving tensor products, which will prove equivalent to the other three:

(4) Given $0 \neq x \in K$, if $I$ and $J$ denote the kernels of $R[X] \rightarrow R[x]$ and $R[Y] \rightarrow R[1 / x]$ respectively ( $X$ and $Y$ indeterminates), then $X Y-1$ $\in(I, J) R[X, Y]$.

$(3) \rightarrow(4)$. Let $x=a / b(a, b \in R)$. Then $b X-a \in I$ and $a Y-b \in J$. Hence $b(X Y-1)=Y(b X-a)+a Y-b \in(I, J) R[X, Y]$. Since $b$ is not a divisor of zero in $R[x] \otimes_{R} R[1 / x]=(R[X] / I) \otimes_{R}(R[Y] / J)$ $=R[X, Y] /(I, J) R[X, Y]$, it follows that $X Y-1 \in(I, J) R[X, Y]$. $(4) \rightarrow(1)$. Since (4) is clearly preserved under localization, it suffices to prove in the quasilocal case that $R$ is a valuation ring. Let 0 $\neq x \in K$. " $X Y-1 \in(I, J) R[X, Y]$ " implies that some polynomial in $I$ or $J$ has a unit constant term, and so that $x$ or $1 / x$ is integrally dependent on $R$. This shows that the integral closure $R^{*}$ of $R$ is a valuation ring; we shall prove that $R=R^{*}$. Let $M$ and $M^{*}$ denote the maximal ideals of $R$ and $R^{*}$ respectively.

(i) $R / M=R^{*} / M^{*}$. Let $x$ be a unit of $R^{*}$ and let $f \in(R / M)[X]$ and $g \in(R / M)[Y]$ denote the minimal polynomials for $\bar{x}$ and $1 / \bar{x}$ over $R / M$. (Here $\bar{x}$ denotes the canonical image of $x$ in $R^{*} / M^{*}$.) From " $X Y-1 \in(I, J) R[X, Y]$ " it follows that

$$
X Y-1 \in(f, g)(R / M)[X, Y] \text {. }
$$


Consequently, upon substituting $\bar{x}$ for $X$ in $\left(R^{*} / M^{*}\right)[X, Y]$, we see that $\bar{x} Y-1$ is divisible in $\left(R^{*} / M^{*}\right)[Y]$ by $g$, and so that $g$ is of degree 1 .

(ii) If $y \in M^{*}$ and $y^{2} \in R$, then $y \in R$. Let $y^{2}=a$ and let $x=1+y$. Note that $x$ is a root of $X^{2}-2 X+1-a$. Therefore, given $f \in I$, $f=\left(X^{2}-2 X+1-a\right) q+r$, where $q \in R[X], r \in I$, and $r$ is of degree at most 1 . Observe that $x, 1 / x$ and $y \in R$ if $r \notin M R[X]$, and consequently that $X Y-1 \in\left(X^{2}-2 X+1-a, J, M\right) R[X, Y]$ if $y \notin R$. In the latter case, reducing $R[X, Y] \bmod M R[X, Y]$ and substituting 1 for $Y$ yields the absurdity that $X-1$ is divisible in $(R / M)[X]$ by $(X-1)^{2}$.

(iii) Given $z \in R^{*}, M$ is not the radical of the conductor of $R[z]$ over $R$. Suppose that $M$ is the radical of the conductor. Then the conductor must be $M$, for otherwise, contrary to (ii), there is a $y \in M^{*}-R$ with $y^{2} \in R$. (Just take $u \in M$, but not in the conductor, and such that $u^{2}$ is in the conductor; choose $v \in R[z]$ such that $u v \notin R$; set $y=u v$.) It follows that $M R[z]=M$ is the maximal ideal of $R[z]$, for the contrary assumption leads directly to a contradiction of (ii). This last fact and (i) together imply that $R=R[z]$, in which case the conductor is $R$.

Now observe that since the prime ideals of $R$ are totally ordered by inclusion - this because the ideals of the valuation ring $R^{*}$ are so ordered-the radical of any proper ideal of $R$ is prime. Supposing $z \in R^{*}-R$, let $P$ be the radical of the conductor of $R[z]$ over $R$. Then the maximal ideal of $R_{P}$ is the radical of the conductor of $R_{P}[z]$ over $R_{P}$. Since (4) is valid for $R_{P}$ as well as for $R$, we have arrived at a contradiction of (iii).

REMARK. The arguments given above generalize "canonically" according to the prescription given in [4] to the context of rings with divisors of zero. The interested reader is invited to check this for himself.

\section{REFERENCES}

1. A. Hattori, On Prüfer rings, J. Math. Soc. Japan 9 (1957), 381-385.

2. F. Richman, Generalized quotient rings, Proc. Amer. Math. Soc. 16 (1965), 794-799.

3. H. Cartan and S. Eilenberg, Homological algebra, Princeton Univ. Press, Princeton, N. J., 1956.

4. E. D. Davis, Overrings of commutative rings. II, Trans. Amer. Math. Soc. 110 (1964), 196-212.

Purdue University 